\title{
A first-class approach of higher derivative Maxwell-Chern-Simons-Proca model
}

\author{
Silviu-Constantin Sararu ${ }^{\mathrm{a}}$ \\ Department of Physics, University of Craiova, 13 A. I. Cuza Str., 200585 Craiova, Romania
}

Received: 6 July 2015 / Accepted: 16 October 2015 / Published online: 5 November 2015

(C) The Author(s) 2015. This article is published with open access at Springerlink.com

\begin{abstract}
The equivalence between a higher derivative extension of Maxwell-Chern-Simons-Proca model and some gauge invariant theories from the point of view of the Hamiltonian path integral quantization in the framework of the gauge-unfixing approach is investigated. The Hamiltonian path integrals of the first-class systems take manifestly Lorentz-covariant forms.
\end{abstract}

\section{Introduction}

The quantization of a second-class constrained system can be achieved by the reformulation of the original theory as a first-class one and then quantizing the resulting first-class theory. This quantization procedure was applied to various models [1-18] using a variety of methods to replace the original second-class model to an equivalent model in which only first-class constraints appear. The conversion of the original second-class system into an equivalent gauge invariant theory can be accomplished without enlarging the phase space, starting from the possibility of interpreting a second-class constraints set as resulting from a gauge-fixing procedure of a first-class constraints one and "undo" gauge-fixing [19-23]. The gauge-unfixing method relies on separating the secondclass constraints into two subsets, one of them being firstclass and the other one providing some canonical gauge conditions for the first-class subset. Starting from the canonical Hamiltonian of the original second-class system, we construct a first-class Hamiltonian with respect to the firstclass subset through an operator that projects any smooth function defined on the phase space into an application that is in strong involution with the first-class subset. Another method to construct the equivalent first-class theory relies on an appropriate extension of the original phase space through the introduction of some new variables. The first-class con-

\footnotetext{
a e-mail: scsararu@central.ucv.ro
}

straints set and the first-class Hamiltonian are constructed as power series in the new variables [24-27]. Various aspects of the equivalence [28] between the self-dual model [29] and the Maxwell-Chern-Simons (MCS) theory [30,31] have been studied using one of the two methods mentioned in the above [17,32-34]. A generalization of the Proca action for a massive vector field with derivative self-interactions in $D=4$ has been constructed in [35]. In [36-40] one finds higher derivative extensions that involve the Maxwell and/or Chern-Simons (CS) terms [28-30]. The Lagrangian of such model is the sum of Maxwell, CS, and higher derivative extensions of these terms. The generalized MCS-Podolsky model $[38,41]$ is a such theory and was introduced in order to smooth ultraviolet singularities. Starting from the observation that the study of Einstein-Chern-Simons-Proca massive gravity (ECSPMG) (the Lagrangian of ECSPMG is the sum of the Einstein, (third derivative order) CS and Procalike mass terms) [42] is often accompanied [39,40,43] by the analysis of the MCS-Proca model (a non-higher derivative model) [37,38,43-45], we consider a model described by Lagrangian action containing the Maxwell term, a higher derivative extension of the CS topological invariant [36], and a Proca mass term

$$
\begin{aligned}
S= & \int d^{3} x\left[-\frac{a}{4} \partial_{[\mu} A_{\nu]} \partial^{[\mu} A^{\nu]}\right. \\
& \left.+\frac{1}{2 b} \varepsilon_{\mu \nu \rho}\left(\partial_{\lambda} \partial^{\lambda} A^{\mu}\right) \partial^{\nu} A^{\rho}-\frac{m^{2}}{2} A_{\mu} A^{\mu}\right]
\end{aligned}
$$

and we investigate from the point of view of the Hamiltonian path integral quantization using the gauge-unfixing (GU) approach the previous higher derivative extension of the MCS-Proca model. The choice of the extended MCSProca (MECS-Proca) model will become more transparent in Sect. 3.1, where we will find that the extended MCS-Proca (MECS-Proca) model and the ECSPMG theory have similarities regarding the number of physical degrees of freedom and the presence of ghosts and tachyon excitations. In order 
to construct an equivalent first-class system starting from the MECS-Proca model in the framework of the GU approach, we need to know the structure of the constraints set of the model. As the second term in the action (1) contains higher derivative terms $\left\{\partial_{\lambda} \partial^{\lambda} A_{\mu}\right\}$, the canonical analysis will be done by a variant of Ostrogradsky method [46-51] developed in Ref. [52], based on an equivalent first order formalism $[53,54]$ and applied to a number of particle and field theoretic models [52,55-57]. The Hamiltonian analysis of a higher derivative extension of a theory displays a constraints set with a more complicated structure than the constraints set of the usual theory (where the Lagrangian is a function of the fields and their first derivatives only). The separation of a second-class constraints set with a complicated structure in two subsets (one of them being first-class and the other one providing some canonical gauge conditions for the firstclass subset) is an intricate issue. In general, in the structure of the constraints set of the higher derivative extension we find a reminiscence of the structure of the constraints set of the usual theory. In order to make the approach of the MECS-Proca model more transparent, initially we consider the MCS-Proca model and we apply the quantization procedure mentioned in the above. Next, we focus on the Hamiltonian analysis of the MECS-Proca model and the construction of the equivalent first-class system using gauge-unfixing method. Then we construct the Hamiltonian path integral of the equivalent first-class system. After integrating out the auxiliary fields and performing some field redefinitions, we discover the manifestly Lorentz-covariant path integral corresponding to the Lagrangian formulation of the first-class system, which reduces to the Lagrangian path integral for a Stückelberg coupling between a scalar field and a 1-form or to the Lagrangian path integral for two kinds of 1-forms with CS coupling.

The paper is organized in four sections. In Sect. 2, starting from MCS-Proca model we construct an equivalent firstclass model using gauge-unfixing method and meanwhile we obtain the path integral corresponding to the first-class system associated with this model. Section 3 contains the main results of the present paper. First, we perform a Hamiltonian analysis and study the excitations and mass counts of the MECS-Proca model. Second, we exemplify in detail the gauge-unfixing method on the MECS-Proca model and then we construct the path integral of the equivalent first-class system associated with this second-class theory. Section 4 ends the paper with the main conclusions.

\section{The MCS-Proca model}

The MCS-Proca model is described by the Lagrangian action [37, 38, 43-45]

$$
\begin{aligned}
S= & \int d^{3} x\left(-\frac{a}{4} \partial_{[\mu} A_{\nu]} \partial^{[\mu} A^{\nu]}\right. \\
& \left.-b \varepsilon_{\mu \nu \rho} A^{\mu} \partial^{\nu} A^{\rho}-\frac{m^{2}}{2} A_{\mu} A^{\mu}\right),
\end{aligned}
$$

where $a$ and $b$ are some real constants. We work with the Minkowski metric tensor of 'mostly minus' signature $\sigma_{\mu \nu}=$ $\operatorname{diag}(+--)$. The canonical analysis $[58,59]$ of the model described by the Lagrangian action (2) displays the secondclass constraints (scc)

$\chi^{(1)} \equiv p^{0} \approx 0$

$\chi^{(2)} \equiv \partial_{i} p^{i}-b \varepsilon^{0 i j} \partial_{i} A_{j}-m^{2} A^{0} \approx 0$,

and the canonical Hamiltonian

$$
\begin{aligned}
H_{c}= & \int d^{2} x\left(-\frac{1}{2 a} p_{i} p^{i}-A_{0} \partial_{i} p^{i}+\frac{a}{4} \partial_{[i} A_{j]} \partial^{[i} A^{j]}\right. \\
& \left.+b \varepsilon_{0 i j} A^{0} \partial^{i} A^{j}+\frac{b}{a} \varepsilon_{0 i j} A^{i} p^{j}-\frac{b^{2}}{2 a} A_{i} A^{i}+\frac{m^{2}}{2} A_{\mu} A^{\mu}\right),
\end{aligned}
$$

where $p^{\mu}$ are the canonical momenta conjugated with the fields $A_{\mu}$. The number of physical degrees of freedom [21] of the original system is equal to

$$
\begin{aligned}
\mathscr{N}_{O} & =(6 \text { canonical variables }-2 \mathrm{scc}) / 2 \\
& =2 .
\end{aligned}
$$

The same result, with respect to the number of degrees of freedom, is obtained in Refs. [43,44]. Moreover, in Refs. $[43,44]$ it is shown that the MCS-Proca model describes a topological mass mix with two massive degrees of freedom, with masses $\sqrt{b^{2}+m^{2}} \pm|b|$.

According to the GU method, we consider the constraint (4) as the first-class constraint (fcc) and the remaining constraint (3) as the corresponding canonical gauge condition. Further, we redefine the first-class constraint

$G \equiv-\frac{1}{m^{2}}\left(\partial_{i} p^{i}-b \varepsilon^{0 i j} \partial_{i} A_{j}-m^{2} A^{0}\right) \approx 0$.

The other choice, considering the constraint (3) as the firstclass constraint and the constraint (4) as the corresponding canonical gauge condition, yields a path integral that cannot be written (after integrating out auxiliary variables) in a manifestly covariant form $[11,14]$. The next step of the GU approach is represented by the construction of a first-class Hamiltonian with respect to the constraint (7),

$$
H_{G U}=H_{c}-\chi^{(1)}\left[G, H_{c}\right]+\frac{1}{2} \chi^{(1)} \chi^{(1)}\left[G,\left[G, H_{c}\right]\right]-\cdots
$$


The concrete form of first-class Hamiltonian, $H_{G U}$ is given by

$$
\begin{aligned}
H_{G U}= & \int d^{2} x\left[-\frac{1}{2 a} p_{i} p^{i}+\frac{a}{4} \partial_{[i} A_{j]} \partial^{[i} A^{j]}\right. \\
& +\frac{b}{a} \varepsilon_{0 i j} A^{i} p^{j}-\frac{b^{2}}{2 a} A_{i} A^{i}-\frac{m^{2}}{2} A_{0} A^{0} \\
& -A_{0}\left(\partial_{i} p^{i}-b \varepsilon^{0 i j} \partial_{i} A_{j}-m^{2} A^{0}\right) \\
& \left.+\frac{1}{2}\left(\frac{1}{m} \partial_{i} p_{0}+m A_{i}\right)\left(\frac{1}{m} \partial^{i} p_{0}+m A^{i}\right)\right] .
\end{aligned}
$$

It can be verified that the Hamiltonian gauge algebra relation is given by

$\left[G, H_{G U}\right]=0$.

The equations of motion are

$$
\begin{aligned}
\dot{A}_{0}= & -\partial_{i}\left(A^{i}+\frac{1}{m^{2}} \partial^{i} p^{0}\right) \\
\dot{A}_{i}= & -\frac{1}{a}\left(p_{i}+b \varepsilon_{0 i j} A^{j}\right)+\partial_{i} A_{0}+\frac{1}{m^{2}} \partial_{i} \Lambda \\
\dot{p}^{0}= & \partial_{i} p^{i}-b \varepsilon^{0 i j} \partial_{i} A_{j}-m^{2} A^{0}-\Lambda \\
\dot{p}^{i}= & a \partial_{j} \partial^{[j} A^{i]}-\frac{b}{a} \varepsilon_{0 i j}\left(p^{j}+b \varepsilon^{0 j k} A_{k}\right) \\
& -m^{2}\left(A^{i}+\frac{1}{m^{2}} \partial^{i} p^{0}\right)-b \varepsilon_{0 i j} \partial^{j} A^{0}-\frac{b}{m^{2}} \varepsilon^{0} i j \partial_{j} \Lambda,
\end{aligned}
$$

where $\Lambda$ is an arbitrary function. Under the canonical gauge condition $p^{0} \approx 0(\Lambda=0)$, Eqs. (11)-(14) return to the equations of motion for the MCS-Proca model. The number of physical degrees of freedom of the GU system is equal to

$$
\begin{aligned}
\mathscr{N}_{G U} & =(6 \text { canonical variables }-2 \times 1 \mathrm{fcc}) / 2 \\
& =2=\mathscr{N}_{O} .
\end{aligned}
$$

The original second-class theory and, respectively, the gaugeunfixed system are classically equivalent since they possess the same number of physical degrees of freedom and, moreover, the corresponding algebras of classical observables are isomorphic. Consequently, the two systems become equivalent at the level of the path integral quantization, which allows us to replace the Hamiltonian path integral of the MCS-Proca model with that of the gauge-unfixed first-class system

$$
\begin{aligned}
Z_{G U}= & \int \mathscr{D}\left(A_{\mu}, p^{\mu}, \lambda\right) \mu\left(\left[A_{\mu}\right]\right) \exp \left\{i \int d ^ { 3 } x \left[\left(\partial_{0} A_{\mu}\right) p^{\mu}\right.\right. \\
& \left.\left.-\mathscr{H}_{G U}+\frac{1}{m^{2}} \lambda\left(\partial^{i} p_{i}-b \varepsilon^{0 i j} \partial_{i} A_{j}-m^{2} A^{0}\right)\right]\right\}
\end{aligned}
$$

where the integration measure ' $\mu\left(\left[A_{\mu}\right]\right)$ ' associated with the model subject to the first-class constraint (7) includes some suitable canonical gauge conditions and it is chosen such that the path integral (16) is convergent [60].

Using in the path integral the notation

$\bar{A}_{0}=A_{0}+\frac{1}{m^{2}} \lambda$

and performing partial integrations over the momentum $p^{i}$ and field $A_{0}$, the argument of the exponential takes the form

$$
\begin{aligned}
S_{G U}= & \int d^{3} x\left[-\frac{a}{4} \partial_{[i} A_{j]} \partial^{[i} A^{j]}-\frac{a}{2}\left(\partial_{0} A_{i}-\partial_{i} \bar{A}_{0}\right)\right. \\
& \times\left(\partial^{0} A^{i}-\partial^{i} \bar{A}^{0}\right)-b \varepsilon_{0 i j} \bar{A}^{0} \partial^{i} A^{j} \\
& -b \varepsilon_{i 0 j} A^{i} \partial^{0} A^{j}-b \varepsilon_{i j 0} A^{i} \partial^{j} \bar{A}^{0} \\
& -\frac{1}{2}\left(\frac{1}{m} \partial_{i} p_{0}+m A_{i}\right)\left(\frac{1}{m} \partial^{i} p^{0}+m A^{i}\right) \\
& \left.-\frac{1}{2}\left(\frac{1}{m} \partial_{0} p_{0}+m \bar{A}_{0}\right)\left(\frac{1}{m} \partial^{0} p^{0}+m \bar{A}^{0}\right)\right] .
\end{aligned}
$$

In terms of the notation $\varphi=-\frac{1}{m} p^{0}$, the last functional reads

$$
\begin{aligned}
S_{G U}= & \int d^{3} x\left[-\frac{a}{4} \partial_{[\mu} \bar{A}_{\nu]} \partial^{[\mu} \bar{A}^{\nu]}-b \varepsilon_{\mu \nu \rho} \bar{A}^{\mu} \partial^{\nu} \bar{A}^{\rho}\right. \\
& \left.-\frac{1}{2}\left(\partial_{\mu} \varphi-m \bar{A}_{\mu}\right)\left(\partial^{\mu} \varphi-m \bar{A}^{\mu}\right)\right],
\end{aligned}
$$

where $\bar{A}_{\mu} \equiv\left\{\bar{A}_{0}, A_{i}\right\}$ and describes a Stückelberg coupling between the scalar field $\varphi$ and the 1-form $\bar{A}_{\mu}$ [61]. The scalar field $\varphi$ plays the role of the Stückelberg scalar. Using the extended phase space method [24-27] in [3-5,9,10] a similar result (for $a=0$ or $b=0$ ) has been obtained. The extra field of the extended phase space method was identified with Stuickelberg scalar. In contrast, in the GU approach we find that to Stuickelberg scalar corresponds $-\frac{1}{m} p^{0}$, where $p^{0}$ is canonical momentum conjugated with the original field $A_{0}$.

In the following we prove that starting from the Hamiltonian path integral of the gauge system (19) with a suitable gauge we recover the MCS-Proca model. The canonical analysis of the model described by the Lagrangian action (2) displays the first-class constraints,

$G_{1} \equiv p^{0} \approx 0, \quad G_{2} \equiv \partial_{i} p^{i}-b \varepsilon^{0 i j} \partial_{i} A_{j}-m p \approx 0$,

and the Hamiltonian

$$
\begin{aligned}
H= & \int d^{2} x\left[-\frac{1}{2 a} p_{i} p^{i}-A_{0} \partial_{i} p^{i}+\frac{a}{4} \partial_{[i} A_{j]} \partial^{[i} A^{j]}\right. \\
& +b \varepsilon_{0 i j} A^{0} \partial^{i} A^{j}+\frac{b}{a} \varepsilon_{0 i j} A^{i} p^{j}-\frac{b^{2}}{2 a} A_{i} A^{i} \\
& \left.-\frac{1}{2} p^{2}+m A^{0} p+\frac{1}{2}\left(\partial_{i} \varphi-m A_{i}\right)\left(\partial^{i} \varphi-m A^{i}\right)\right],
\end{aligned}
$$


where $\left\{p^{\mu}, p\right\}$ are the canonical momenta conjugate with the fields $\left\{A_{\mu}, \varphi\right\}$. Taking

$C^{1} \equiv \varphi \approx 0, \quad C^{2} \equiv-p+m A_{0} \approx 0$,

as the unitary gauge-fixing conditions, the Hamiltonian path integral is given by

$$
\begin{aligned}
Z= & \int \mathscr{D}\left(A_{\mu}, p^{\mu}, \varphi, p\right) \delta\left(G_{1}\right) \delta\left(G_{2}\right) \delta\left(C^{1}\right) \delta\left(C^{2}\right) \\
& \times \exp \left\{i \int d^{3} x\left[\left(\partial_{0} A_{\mu}\right) p^{\mu}+\left(\partial_{0} \varphi\right) p-\mathscr{H}\right]\right\} .
\end{aligned}
$$

Integrating over the momentum $p^{0}$ and fields $\left\{\varphi, A_{0}\right\}$ and representing $\delta\left(\partial_{i} p^{i}-b \varepsilon^{0 i j} \partial_{i} A_{j}-m p\right)$ in the form of an integral functional,

$$
\int \mathscr{D} \lambda \exp \left\{i \int d^{3} x\left[\lambda\left(\partial_{i} p^{i}-b \varepsilon^{0 i j} \partial_{i} A_{j}-m p\right)\right]\right\},
$$

the path integral takes the form

$$
\begin{aligned}
Z= & \int \mathscr{D}\left(A_{i}, p^{i}, p, \lambda\right) \exp \left\{i \int d ^ { 3 } x \left[\left(\partial_{0} A_{i}\right) p^{i}\right.\right. \\
& +\frac{1}{2 a} p_{i} p^{i}+\frac{1}{m} p \partial_{i} p^{i}-\frac{a}{4} \partial_{[i} A_{j]} \partial^{[i} A^{j]} \\
& -\frac{b}{m} \varepsilon_{0 i j} p \partial^{i} A^{j}-\frac{b}{a} \varepsilon_{0 i j} A^{i} p^{j}+\frac{b^{2}}{2 a} A_{i} A^{i}-\frac{1}{2} p^{2} \\
& \left.\left.-\frac{m^{2}}{2} A_{i} A^{i}+\lambda\left(\partial_{i} p^{i}-b \varepsilon^{0 i j} \partial_{i} A_{j}-m p\right)\right]\right\} .
\end{aligned}
$$

Performing in the path integral the notation

$A_{0}=\frac{1}{m} p+\lambda$,

the argument of the exponential becomes

$$
\begin{aligned}
Z= & \int \mathscr{D}\left(A_{\mu}, p^{i}, p\right) \exp \left\{i \int d ^ { 3 } x \left[\left(\partial_{0} A_{i}\right) p^{i}\right.\right. \\
& +\frac{1}{2 a} p_{i} p^{i}-\frac{a}{4} \partial_{[i} A_{j]} \partial^{[i} A^{j]}-\frac{b}{a} \varepsilon_{0 i j} A^{i} p^{j} \\
& -\frac{b^{2}}{2 a} A_{i} A^{i}+\frac{1}{2} p^{2}-\frac{m^{2}}{2} A_{i} A^{i}+A_{0} \\
& \left.\left.\times\left(\partial_{i} p^{i}-b \varepsilon^{0 i j} \partial_{i} A_{j}-m p\right)\right]\right\} .
\end{aligned}
$$

After integration over the momenta $p^{i}$ and $p$, we find that the argument of the exponential is just the MCS-Proca Lagrangian

$$
\begin{aligned}
Z= & \int \mathscr{D} A_{\mu} \exp \left\{i \int d ^ { 3 } x \left(-\frac{a}{4} \partial_{[\mu} A_{\nu]} \partial^{[\mu} A^{\nu]}\right.\right. \\
& \left.\left.-b \varepsilon_{\mu \nu \rho} A^{\mu} \partial^{\nu} A^{\rho}-\frac{m^{2}}{2} A_{\mu} A^{\mu}\right)\right\} .
\end{aligned}
$$

The MCS-Proca model can be correlated to another firstclass theory whose field spectrum comprise two types of 1form gauge fields. For this purpose we consider the following fields/momenta combinations:

$\mathscr{P}_{i} \equiv p_{i}+b \varepsilon_{0 i j} A^{j}, \quad \mathscr{F}_{i} \equiv A_{i}+\frac{1}{m^{2}} \partial_{i} p_{0}, \quad \mathscr{F}_{0} \equiv A_{0}$,

which are in (strong) involution with the first-class constraint (7),

$\left[\mathscr{P}_{i}, G\right]=\left[\mathscr{F}_{i}, G\right]=\left[\mathscr{F}_{0}, G\right]=0$.

We observe that the first-class Hamiltonian (9) can be written in terms of these gauge invariant quantities as

$$
\begin{aligned}
H_{G U}= & \int d^{2} x\left[-\frac{1}{2 a} \mathscr{P}_{i} \mathscr{P}^{i}+\frac{a}{4} \partial_{[i} \mathscr{F}_{j]} \partial^{[i} \mathscr{F}^{j]}\right] \\
& \left.+\frac{m^{2}}{2} \mathscr{F}_{i} \mathscr{F}^{i}-\frac{m^{2}}{2} \mathscr{F}_{0} \mathscr{F}^{0}+m^{2} \mathscr{F}_{0} G\right] .
\end{aligned}
$$

By direct computation we find that $\mathscr{F}_{\mu} \equiv\left\{\mathscr{F}_{0}, \mathscr{F}_{i}\right\}$ satisfy the equations

$\partial^{\nu} \partial_{[\nu} \mathscr{F}_{0]}=\frac{m^{2}}{a} \mathscr{F}_{0}+\frac{2 b}{a} \varepsilon_{0 i j} \partial^{i} \mathscr{F}^{j}+\mathscr{O}(G)$,

$\partial^{\nu} \partial_{[v} \mathscr{F}_{i]}=\frac{m^{2}}{a} \mathscr{F}_{i}+\frac{2 b}{a^{2}} \varepsilon_{0 i j} \mathscr{P}^{j}+\mathscr{O}(G)$,

and that it is divergenceless,

$\partial^{\mu} \mathscr{F}_{\mu}=0$.

Enlarging the phase space by adding some bosonic canonical variables $\left\{V^{\mu}, P_{\mu}\right\}$, we can write the solution to the Eq. (34) as

$\mathscr{F}_{\mu}=-\frac{1}{m} \varepsilon_{\mu \nu \rho} \partial^{\nu} V^{\rho}$.

When we replace the solution (35) in the first-class constraint (7), the constraint takes the form

$$
-\frac{1}{m^{2}}\left(\partial^{i} p_{i}-b \varepsilon_{0 i j} \partial^{i} A^{j}+m \varepsilon_{0 i j} \partial^{i} V^{j}\right) \approx 0,
$$

and remains first-class. From the gauge transformation of the quantity $\partial_{i} p_{0}$, we obtain

$\partial_{i} p_{0}=m \varepsilon_{0 i j} P^{j}$.

Using Eqs. (35) and (37) in the first-class Hamiltonian (9), we obtain for the first-class Hamiltonian the following form:

$$
\begin{aligned}
H_{G U}^{\prime}= & \int d^{2} x\left[\frac{a}{4} \partial_{[i} A_{j]} \partial^{[i} A^{j]}-\frac{1}{4} \partial_{[i} V_{j]} \partial^{[i} V^{j]}\right. \\
& -\frac{1}{2 a}\left(p_{i}+b \varepsilon_{0 i j} A^{j}\right)\left(p^{i}+b \varepsilon^{0 i k} A_{k}\right)
\end{aligned}
$$




$$
\begin{aligned}
& +\frac{m^{2}}{2}\left(A_{i}+\frac{1}{m} \varepsilon_{0 i j} P^{j}\right)\left(A_{i}+\frac{1}{m} \varepsilon^{0 i k} P_{k}\right) \\
& +\frac{1}{2 m} \varepsilon_{0 i j} \partial^{[i} V^{j]} \\
& \left.\times\left(\partial_{k} p^{k}-b \varepsilon_{0 k l} \partial^{k} A^{l}+m \varepsilon_{0 k l} \partial^{k} V^{l}\right)\right] .
\end{aligned}
$$

In this moment we have a dynamical system with the phase space locally parameterized by $\left\{A_{i}, p^{i}, V^{\mu}, P_{\mu}\right\}$, subject to the first-class constraint (36) and too many degrees of freedom

$$
\begin{aligned}
\mathscr{N}_{G U}^{\prime} & =(10 \text { canonical variables }-2 \times 1 \mathrm{fcc}) / 2 \\
& =4 \neq \mathscr{N}_{G U} .
\end{aligned}
$$

In order to cut the two extra degrees of freedom, we impose in addition to the first-class constraint (36) two supplementary first-class constraints,

$-\partial_{i} P^{i} \approx 0, \quad P^{0} \approx 0$,

and we obtain a first-class system with a right number of physical degrees of freedom,

$$
\begin{aligned}
\mathscr{N}_{G U}^{\prime} & =(10 \text { canonical variables }-2 \times 3 \mathrm{fcc}) / 2 \\
& =2=\mathscr{N}_{G U} .
\end{aligned}
$$

Since the number of physical degrees of freedom is the same for both first-class theories and for each of them we are able to identify a set of fundamental classical observables such that they are in one-to-one correspondence and possess the same Poisson brackets, the first-class theories are equivalent. As a result, the GU and the first-class systems remain equivalent also at the level of the Hamiltonian path integral quantization. This further implies that the first-class system is completely equivalent with the original second-class theory. Due to this equivalence we can replace the Hamiltonian path integral of MCS-Proca model with the one associated with the first-class system,

$$
\begin{aligned}
Z^{\prime}= & \int \mathscr{D}\left(A_{i}, V^{\mu}, p^{i}, P_{\mu}, \lambda^{\prime} s\right) \mu\left(\left[A_{i}\right],\left[V^{\mu}\right]\right) \\
& \times \exp \left\{i \int d ^ { 3 } x \left[\left(\partial_{0} A_{i}\right) p^{i}+\left(\partial_{0} V^{\mu}\right) P_{\mu}\right.\right. \\
& -\mathscr{H}_{G U}^{\prime}+\lambda^{(1)} \partial_{i} P^{i}-\lambda^{(2)} P^{0} \\
& \left.\left.+\frac{1}{m^{2}} \lambda\left(\partial_{i} p^{i}-b \varepsilon_{0 i j} \partial^{i} A^{j}+m \varepsilon_{0 i j} \partial^{i} V^{j}\right)\right]\right\} .
\end{aligned}
$$

If we perform in the path integral the partial integrations over $\left\{V^{0}, p_{i}, P_{\mu}, \lambda^{(2)}\right\}$ and use the notations

$$
\bar{A}_{0}=\frac{1}{m^{2}}\left(\lambda-\frac{m}{2} \varepsilon_{0 i j} \partial^{[i} V^{j]}\right), \quad \bar{V}_{0}=\lambda^{(1)},
$$

the argument of the exponential becomes

$$
\begin{aligned}
S_{G U}^{\prime}= & \int d^{3} x\left[-\frac{a}{4} \partial_{[i} A_{j]} \partial^{[i} A^{j]}\right. \\
& -\frac{a}{2}\left(\partial_{0} A_{i}-\partial_{i} \bar{A}_{0}\right)\left(\partial^{0} A^{i}-\partial^{i} \bar{A}^{0}\right) \\
& -b \varepsilon_{0 i j} \bar{A}^{0} \partial^{i} A^{j}-b \varepsilon_{i 0 j} A^{i} \partial^{0} A^{j}-b \varepsilon_{i j 0} A^{i} \partial^{j} \bar{A}^{0} \\
& +\frac{1}{4} \partial_{[i} V_{j]} \partial^{[i} V^{j]}+\frac{1}{2}\left(\partial_{0} V_{i}-\partial_{i} \bar{V}_{0}\right)\left(\partial^{0} V^{i}-\partial^{i} \bar{V}^{0}\right) \\
& \left.+m \varepsilon_{0 i j} \bar{A}^{0} \partial^{i} V^{j}+m \varepsilon_{i 0 j} A^{i} \partial^{0} V^{j}+m \varepsilon_{i j 0} A^{i} \partial^{j} \bar{V}^{0}\right] .
\end{aligned}
$$

The argument of the exponential takes a manifestly Lorentzcovariant form

$$
\begin{aligned}
S_{G U}^{\prime}= & \int d^{3} x\left(-\frac{a}{4} \partial_{[\mu} \bar{A}_{\nu]} \partial^{[\mu} \bar{A}^{\nu]}-b \varepsilon_{\mu \nu \rho} \bar{A}^{\mu} \partial^{\nu} \bar{A}^{\rho}\right. \\
& \left.+\frac{1}{4} \partial_{[\mu} \bar{V}_{\nu]} \partial^{[\mu} \bar{V}^{\nu]}+m \varepsilon_{\mu \nu \rho} \bar{A}^{\mu} \partial^{\nu} \bar{V}^{\rho}\right),
\end{aligned}
$$

where $\bar{A}_{\mu} \equiv\left\{\bar{A}_{0}, A_{i}\right\}$ and $\bar{V}_{\mu} \equiv\left\{\bar{V}_{0}, V_{i}\right\}$. The functional (45) associated with the first-class system describes a CS coupling between the two 1 -forms, $\bar{A}_{\mu}$ and $\bar{V}_{\mu}$ [62].

\section{The higher derivative MCS-Proca model}

\subsection{Hamiltonian analysis of the MECS-Proca model}

The starting point of the approach developed in [52] consists in converting the original higher derivative theory to an equivalent first order theory by introducing new fields to account for higher derivative terms. To pass from the higher derivative theory to a first order one, we define the variables $B_{\mu}$ as

$B_{\mu}=\partial_{0} A_{\mu}$,

and enforce the Lagrangian constraints

$B_{\mu}-\partial_{0} A_{\mu}=0$

by the Lagrange multiplier $\xi^{\mu}$

$$
\begin{aligned}
\mathscr{L}= & -\frac{a}{4} \partial_{[i} A_{j]} \partial^{[i} A^{j]}-\frac{a}{2}\left(B_{i}-\partial_{i} A_{0}\right)\left(B^{i}-\partial^{i} A^{0}\right) \\
& +\frac{1}{2 b} \varepsilon_{0 i j}\left(\partial_{0} B^{0}+\partial_{k} \partial^{k} A^{0}\right) \partial^{i} A^{j} \\
& +\frac{1}{2 b} \varepsilon_{i 0 j}\left(\partial_{0} B^{i}+\partial_{k} \partial^{k} A^{i}\right) B^{j} \\
& +\frac{1}{2 b} \varepsilon_{i j 0}\left(\partial_{0} B^{i}+\partial_{k} \partial^{k} A^{i}\right) \partial^{j} A^{0} \\
& -\frac{m^{2}}{2} A_{\mu} A^{\mu}+\xi^{\mu}\left(B_{\mu}-\partial_{0} A_{\mu}\right)
\end{aligned}
$$


From the definitions of the canonical momenta $\left\{\Pi_{\mu}, p^{\mu}, \pi^{\mu}\right\}$ conjugate to the fields $\left\{\xi^{\mu}, A_{\mu}, B_{\mu}\right\}$

$\Pi_{\mu}=\frac{\partial L}{\partial \dot{\xi}^{\mu}}, \quad p^{\mu}=\frac{\partial L}{\partial \dot{A}_{\mu}}, \quad \pi^{\mu}=\frac{\partial L}{\partial \dot{B}_{\mu}}$,

we obtain the primary constraints,

$\Phi_{\mu}^{(\xi)} \equiv \Pi_{\mu} \approx 0$,

$\Phi^{(A) \mu} \equiv p^{\mu}+\xi^{\mu} \approx 0$,

$\Phi_{i}^{(B)} \equiv \pi_{i}+\frac{1}{2 b} \varepsilon_{0 i j}\left(B^{j}-\partial^{j} A^{0}\right) \approx 0$,

$\Phi^{(B)} \equiv \pi_{0}-\frac{1}{2 b} \varepsilon_{0 i j} \partial^{i} A^{j} \approx 0$.

If we write the primary constraints (52)-(53) in an equivalent form

$\Phi_{i}^{(B)} \equiv \pi_{i}+\frac{1}{2 b} \varepsilon_{0 i j}\left(B^{j}-\partial^{j} A^{0}\right)-\frac{1}{2 b} \varepsilon_{0 i j} \partial^{j} \Pi^{0} \approx 0$,

$\Phi^{\prime(B)} \equiv \pi_{0}-\frac{1}{2 b} \varepsilon_{0 i j} \partial^{i} A^{j}-\frac{1}{2 b} \varepsilon_{0 i j} \partial^{i} \Pi^{j} \approx 0$,

the nonvanishing elements of the algebra of the primary constraints (pc) are

$\left[\Phi_{\mu}^{(\xi)}(x), \Phi^{(A) v}(y)\right]_{x_{0}=y_{0}}=-\delta_{\mu}^{v} \delta^{2}(\mathbf{x}-\mathbf{y})$,

$\left[\Phi_{i}^{(B)}(x), \Phi_{j}^{\prime(B)}(y)\right]_{x_{0}=y_{0}}=\frac{1}{b} \varepsilon_{0 i j} \delta^{2}(\mathbf{x}-\mathbf{y})$.

The canonical Hamiltonian is given by

$$
\begin{aligned}
H_{c}= & \left.\int d^{2} x\left(\Pi_{\mu} \dot{\xi}^{\mu}+p^{\mu} \dot{A}_{\mu}+\pi^{\mu} \dot{B}_{\mu}-\mathscr{L}\right)\right|_{\{p c\}} \\
= & \int d^{2} x\left[\frac{a}{4} \partial_{[i} A_{j]} \partial^{[i} A^{j]}+\frac{a}{2}\left(B_{i}-\partial_{i} A_{0}\right)\left(B^{i}-\partial^{i} A^{0}\right)\right. \\
& -\frac{1}{2 b} \varepsilon_{0 i j}\left(\partial_{k} \partial^{k} A^{0}\right) \partial^{i} A^{j}-\frac{1}{2 b} \varepsilon_{i 0 j}\left(\partial_{k} \partial^{k} A^{i}\right) B^{j} \\
& \left.-\frac{1}{2 b} \varepsilon_{i j 0}\left(\partial_{k} \partial^{k} A^{i}\right) \partial^{j} A^{0}-\xi^{\mu} B_{\mu}+\frac{m^{2}}{2} A_{\mu} A^{\mu}\right],
\end{aligned}
$$

and the total Hamiltonian is

$$
\begin{aligned}
H_{T}= & H_{c}+\int d^{2} x\left(u^{(\xi) \mu} \Phi_{\mu}^{(\xi)}+u_{\mu}^{(A)} \Phi^{(A) \mu}\right. \\
& \left.+u^{(B) i} \Phi_{i}^{(B)}+u^{(B)} \Phi^{\prime(B)}\right),
\end{aligned}
$$

where $\left\{u^{(\xi) \mu}, u_{\mu}^{(A)}, u^{(B) i}, u^{(B)}\right\}$ are Lagrange multipliers.

The consistency of the primary constraints (50), (51), (54) leads to the determination of the Lagrange multipliers $\left\{u^{(\xi) \mu}, u_{\mu}^{(A)}, u^{(B) i}\right\}$, while the consistency of the remaining primary constraint $\Phi^{\prime(B)} \approx 0$ generate the secondary constraint,

$\Phi_{I I}^{(B)} \equiv \xi_{0}-\frac{1}{2 b} \varepsilon_{0 i j} \partial^{i} B^{j} \approx 0$.

The consistency of the secondary constraint yields the tertiary constraint,

$\Phi_{I I I}^{(B)} \equiv \partial_{i} \xi^{i}+m^{2} A_{0}-\frac{1}{2 b} \varepsilon_{0 i j} \partial_{k} \partial^{k} \partial^{i} A^{j} \approx 0$.

Conserving the constraint $\Phi_{I I I}^{(B)} \approx 0$ we get the quartic constraint,

$\Phi_{I V}^{(B)} \equiv m^{2} \partial_{i} A^{i}+m^{2} B_{0} \approx 0$.

The consistency condition of the quartic constraint $\Phi_{I V}^{(B)} \approx 0$ determines the multiplier $u^{(B)}$ and no more new constraint is produced.

The constraints (50), (51), (54), (55), and (60)-(62) are second-class and irreducible. The nonzero Poisson brackets among the constraints functions read

$\left[\Phi_{\mu}^{(\xi)}(x), \Phi^{(A) v}(y)\right]_{x_{0}=y_{0}}=-\delta_{\mu}^{v} \delta^{2}(\mathbf{x}-\mathbf{y})$,

$\left[\Phi_{\mu}^{(\xi)}(x), \Phi_{I I}^{(B)}(y)\right]_{x_{0}=y_{0}}=-\delta_{\mu}^{0} \delta^{2}(\mathbf{x}-\mathbf{y})$,

$\left[\Phi_{\mu}^{(\xi)}(x), \Phi_{I I I}^{(B)}(y)\right]_{x_{0}=y_{0}}=\delta_{\mu}^{i} \partial_{i} \delta^{2}(\mathbf{x}-\mathbf{y})$,

$\left[\Phi^{(A) 0}(x), \Phi_{I I I}^{(B)}(y)\right]_{x_{0}=y_{0}}=-m^{2} \delta^{2}(\mathbf{x}-\mathbf{y})$,

$\left[\Phi^{(A) i}(x), \Phi_{I I I}^{(B)}(y)\right]_{x_{0}=y_{0}}=\frac{1}{2 b} \varepsilon^{0 i j} \partial_{k} \partial^{k} \partial_{j} \delta^{2}(\mathbf{x}-\mathbf{y})$,

$\left[\Phi^{(A) \mu}(x), \Phi_{I V}^{(B)}(y)\right]_{x_{0}=y_{0}}=m^{2} \delta_{i}^{\mu} \partial^{i} \delta^{2}(\mathbf{x}-\mathbf{y})$,

$\left[\Phi^{\prime(B)}(x), \Phi_{I V}^{(B)}(y)\right]_{x_{0}=y_{0}}=-m^{2} \delta^{2}(\mathbf{x}-\mathbf{y})$,

$\left[\Phi_{i}^{\prime(B)}(x), \Phi_{j}^{\prime(B)}(y)\right]_{x_{0}=y_{0}}=\frac{1}{b} \varepsilon_{0 i j} \delta^{2}(\mathbf{x}-\mathbf{y})$,

$\left[\Phi_{i}^{(B)}(x), \Phi_{I I}^{(B)}(y)\right]_{x_{0}=y_{0}}=\frac{1}{b} \varepsilon_{0 i j} \partial^{j} \delta^{2}(\mathbf{x}-\mathbf{y})$.

The number of physical degrees of freedom of the original system is equal to

$\overline{\mathscr{N}}_{O}=(18$ canonical variables $-12 \mathrm{scc}) / 2$

We notice that the number of physical degrees of freedom of the extended model is higher than the number of physical degrees of freedom of the MCS-Proca model,

$\overline{\mathscr{N}}_{O}>\mathscr{N}_{O}$

This result was expected due to the higher derivative nature of the MECS-Proca model. In addition the number of physical 
degrees of freedom of MECS-Proca model coincides with that of the ECSPMG theory.

The analyze of the excitations and mass counts of the MECS-Proca model reveal the fact that if the sign of the Maxwell term is the usual one then the excitation masses will be complex, with the wrong sign the reality of the excitation masses will be restored for a known condition satisfied by parameters $b$ and $m$, but the model faces ghost problems. The action (1) can be rewritten in terms of the transverse operator $\theta_{\mu \nu}=\sigma_{\mu \nu}-\frac{\partial_{\mu} \partial_{\nu}}{\square}$, longitudinal operators $\omega_{\mu \nu}=\frac{\partial_{\mu} \partial_{\nu}}{\square}$ and the operator associated with the topological term $S_{\mu \nu}=\varepsilon_{\mu \rho \nu} \partial^{\rho}$ like

$S=\int d^{3} x \frac{1}{2} A^{\mu} \mathscr{O}_{\mu \nu} A^{v}$,

where $\mathscr{O}_{\mu \nu}=\left(a \square-m^{2}\right) \theta_{\mu \nu}-m^{2} \omega_{\mu \nu}+\frac{1}{b} \square S_{\mu \nu}$. The propagator in the momentum space for the MECS-Proca model is

$$
\begin{aligned}
\mathscr{P}_{\mu \nu}= & -\frac{a k^{2}+m^{2}}{\left(a k^{2}+m^{2}\right)^{2}-\frac{1}{b^{2}} k^{6}} \theta_{\mu \nu} \\
& -\frac{1}{m^{2}} \omega_{\mu \nu}+\frac{\frac{1}{b} k^{2}}{\left(a k^{2}+m^{2}\right)^{2}-\frac{1}{b^{2}} k^{6}} S_{\mu \nu} .
\end{aligned}
$$

Taking into consideration that only the $\theta$-component of the propagator,

$\mathscr{P}^{(\theta)}=-\frac{a k^{2}+m^{2}}{\left(a k^{2}+m^{2}\right)^{2}-\frac{1}{b^{2}} k^{6}}$,

contributes to the current-current transition amplitude, we study the residues at each simple pole of the $\mathscr{P}^{(\theta)}[39,40]$.

We analyze the roots of the cubic equation

$-\frac{1}{b^{2}}\left(k^{2}\right)^{3}+a^{2}\left(k^{2}\right)^{2}+2 a m^{2} k^{2}+m^{4}=0$,

whose discriminant is

$D=4 \frac{m^{8}}{b^{4}}\left(-a^{3} \frac{b^{2}}{m^{2}}-\frac{27}{4}\right)$.

For $a=1$ (Maxwell's term with the usual sign) the discriminant is less than zero and the equation has one real root and two complex conjugate roots. Also, for $a=-1$ (Maxwell's term with the wrong sign) the roots of Eq. (77) are complex unless $\frac{b^{2}}{m^{2}} \geq \frac{27}{4}$. In the limit case $\frac{b^{2}}{m^{2}}=\frac{27}{4}$ the roots coalesce and are

$k_{1}^{2}=k_{2}^{2}=4 k_{3}^{2}=3 m^{2}$.

Therefore, if $a=-1$ and $\frac{b^{2}}{m^{2}}>\frac{27}{4}$ the equation has three distinct real roots. In [42] (see also [43]) Eq. (77) for $a=$ -1 was obtained from the pole propagator of the ECSPMG model, where it was noted that if $\frac{b^{2}}{m^{2}}>\frac{27}{4}$ then the three distinct real roots are all positive. The absence of the tachyons in a theory is provided by the existence of only positive poles, and consequently the MECS-Proca model is free from the tachyons for $a=-1$ and $\frac{b^{2}}{m^{2}}>\frac{27}{4}$. After the analyze of the signs of the residues at each simple pole of $\theta$-component of the propagator, we see that not all residues have the same sign. The signs of the residues at each simple pole of $\theta$-component of the propagator tell us whether the ghosts excitations arise and therefore the MECS-Proca model is plagued by ghosts. We notice that the same problems from the ECSPMG theory about the presence of ghosts and tachyon excitations are also present here, in the MECS-Proca model.

\subsection{The construction of the first-class system}

Imposing the requirement of the constraints (50)-(51) to be strongly zero and eliminating the unphysical sector $\left\{\xi^{\mu}, \Pi_{\mu}\right\}$, the reduced phase space being locally parameterized by $\left\{A_{\mu}, B_{\mu}, p^{\mu}, \pi^{\mu}\right\}$, we arrive at a system subject to the second-class constraints,

$\chi_{i}^{(1)} \equiv \pi_{i}+\frac{1}{2 b} \varepsilon_{0 i j}\left(B^{j}-\partial^{j} A^{0}\right) \approx 0$,

$\chi^{(1)} \equiv \pi_{0}-\frac{1}{2 b} \varepsilon_{0 i j} \partial^{i} A^{j} \approx 0$,

$\chi^{(2)} \equiv-p_{0}-\frac{1}{2 b} \varepsilon_{0 i j} \partial^{i} B^{j} \approx 0$,

$\chi^{(3)} \equiv-\partial_{i} p^{i}+m^{2} A_{0}-\frac{1}{2 b} \varepsilon_{0 i j} \partial_{k} \partial^{k} \partial^{i} A^{j} \approx 0$,

$\chi^{(4)} \equiv m^{2} \partial_{i} A^{i}+m^{2} B_{0} \approx 0$,

while the canonical Hamiltonian (58) takes the form

$$
\begin{aligned}
H_{c}= & \int d^{2} x\left[\frac{a}{4} \partial_{[i} A_{j]} \partial^{[i} A^{j]}+\frac{a}{2}\left(B_{i}-\partial_{i} A_{0}\right)\left(B^{i}-\partial^{i} A^{0}\right)\right. \\
& -\frac{1}{2 b} \varepsilon_{0 i j}\left(\partial_{k} \partial^{k} A^{0}\right) \partial^{i} A^{j}-\frac{1}{2 b} \varepsilon_{i 0 j}\left(\partial_{k} \partial^{k} A^{i}\right) B^{j} \\
& \left.-\frac{1}{2 b} \varepsilon_{i j 0}\left(\partial_{k} \partial^{k} A^{i}\right) \partial^{j} A^{0}+p^{\mu} B_{\mu}+\frac{m^{2}}{2} A_{\mu} A^{\mu}\right] .
\end{aligned}
$$

The nontrivial Poisson brackets between the constraint functions are listed below:

$\left[\chi_{i}^{(1)}(x), \chi_{j}^{(1)}(y)\right]_{x_{0}=y_{0}}=\frac{1}{b} \varepsilon_{0 i j} \delta^{2}(\mathbf{x}-\mathbf{y})$,

$\left[\chi_{i}^{(1)}(x), \chi^{(2)}(y)\right]_{x_{0}=y_{0}}=\frac{1}{b} \varepsilon_{0 i j} \partial^{j} \delta^{2}(\mathbf{x}-\mathbf{y})$,

$\left[\chi^{(1)}(x), \chi^{(4)}(y)\right]_{x_{0}=y_{0}}=-m^{2} \delta^{2}(\mathbf{x}-\mathbf{y})$,

$\left[\chi^{(2)}(x), \chi^{(3)}(y)\right]_{x_{0}=y_{0}}=m^{2} \delta^{2}(\mathbf{x}-\mathbf{y})$,

$\left[\chi^{(3)}(x), \chi^{(4)}(y)\right]_{x_{0}=y_{0}}=-m^{2} \partial_{k} \partial^{k} \delta^{2}(\mathbf{x}-\mathbf{y})$. 
If we make the linear combination of the constraints $\chi^{(2)} \approx 0$ and $\chi_{i}^{(1)} \approx 0$

$\bar{\chi}^{(2)}=\chi^{(2)}+\partial^{i} \chi_{i}^{(1)} \approx 0$,

the matrix of the Poisson bracket among the constraints functions becomes

$C_{\alpha_{0} \beta_{0}}=\left(\begin{array}{ccccc}\frac{1}{b} \varepsilon_{0 i j} & \mathbf{0} & \mathbf{0} & \mathbf{0} & \mathbf{0} \\ \mathbf{0} & 0 & 0 & 0 & -m^{2} \\ \mathbf{0} & 0 & 0 & m^{2} & 0 \\ \mathbf{0} & 0 & -m^{2} & 0 & -m^{2} \partial_{k} \partial^{k} \\ \mathbf{0} & m^{2} & 0 & m^{2} \partial_{k} \partial^{k} & 0\end{array}\right)$.

We notice that the constraints $\chi_{i}^{(1)} \approx 0$ generate a submatrix (of the matrix of the Poisson brackets among the constraints functions) of maximum rank, therefore they form an independent subset of second-class constraints. Thus in the sequel we examine from the point of view of the GU method only the constraints $\chi_{A} \equiv\left\{\chi^{(1)}, \bar{\chi}^{(2)}, \chi^{(3)}, \chi^{(4)}\right\} \approx 0$.

The second-class constraints set $\chi_{A} \approx 0$ cannot be straightforwardly separated in two subsets such that one of them being first-class and the other providing some canonical gauge conditions for the first-class subset. To make this possible, we write the constraints set in an equivalent form

$\chi_{A}^{\prime}=E_{A B} \chi_{B}$,

where $E_{A B}$ is an invertible matrix

$E_{A B}=\left(\begin{array}{cccc}\frac{\partial_{k} \partial^{k}}{m^{2}} & 0 & -\frac{1}{m^{2}} & 0 \\ 0 & 1 & 0 & 0 \\ -1 & 0 & 0 & 0 \\ 0 & 0 & 0 & \frac{1}{m^{2}}\end{array}\right)$.

The concrete form of the constraints $\chi_{A}^{\prime} \approx 0$ is

$\chi^{\prime(1)} \equiv \frac{1}{m^{2}}\left(\partial_{i} p^{i}-m^{2} A_{0}+\partial_{k} \partial^{k} \pi_{0}\right) \approx 0$,

$\chi^{\prime(2)} \equiv-p_{0}+\partial_{i} \pi^{i} \approx 0$,

$\chi^{\prime(3)} \equiv-\pi_{0}+\frac{1}{2 b} \varepsilon_{0 i j} \partial^{i} A^{j} \approx 0$,

$\chi^{\prime(4)} \equiv \partial_{i} A^{i}+B_{0} \approx 0$,

with the matrix of the Poisson brackets among the constraint functions expressed by

$C_{A B}=\left(\begin{array}{cccc}0 & 1 & 0 & 0 \\ -1 & 0 & 0 & 0 \\ 0 & 0 & 0 & 1 \\ 0 & 0 & -1 & 0\end{array}\right)$

Examining the structure of the constraints set (95)-(98) we notice that, in the constraints $\chi^{\prime(1)} \approx 0$ and $\chi^{\prime(2)} \approx 0$, we find a reminiscence of the structure of the constraint set of the MCS-Proca model (3)-(4), while the constraints $\chi^{\prime(3)} \approx 0$ and $\chi^{\prime(4)} \approx 0$ have no counterparts. It was proved in Ref. [20] that for a dynamical system subject to the second-class constraints $\left\{\chi_{\alpha_{0}} \approx 0\right\}_{\alpha_{0}=\overline{1,2 M_{0}}}$, the subsets $\left\{\chi_{1}, \chi_{2}, \ldots, \chi_{M_{0}}\right\}$ and $\left\{\chi_{1}, \chi_{2}, \ldots, \chi_{M_{0}-1}, \chi_{M_{0}+1}\right\}$ of the full set of constraints are first-class sets on $\Sigma_{2 M_{0}}$. According to the above, we consider the subset $G_{a} \equiv\left\{\chi^{\prime(1)}, \chi^{\prime(3)}\right\}$ as the first-class constraint set and the remaining constraints, $C_{a} \equiv\left\{\chi^{\prime(2)}, \chi^{\prime(4)}\right\}$, as the corresponding canonical gauge conditions.

Starting from the canonical Hamiltonian of the original second-class system we construct a first-class Hamiltonian with respect to the first-class subset in two steps [22]. First, we construct the first-class Hamiltonian with respect to the constraint $G_{1} \approx 0$

$$
\begin{aligned}
H_{G U}^{1}= & H_{c}-C_{1}\left[G_{1}, H_{c}\right]+\frac{1}{2} C_{1} C_{1}\left[G_{1}\left[G_{1}, H_{c}\right]\right]-\cdots \\
= & H_{c}+\int d^{2} x\left[\left(-p_{0}+\partial_{i} \pi^{i}\right)\left(\partial_{k} A^{k}+B_{0}\right)\right. \\
& +\frac{1}{m^{2}}\left(-p_{0}+\partial_{i} \pi^{i}\right) \partial_{k} \partial^{k}\left(p_{0}+\frac{1}{2 b} \varepsilon_{0 l m} \partial^{l} B^{m}\right) \\
& \left.+\frac{1}{2 m^{2}}\left(-p_{0}+\partial_{i} \pi^{i}\right) \partial_{k} \partial^{k}\left(-p_{0}+\partial_{j} \pi^{j}\right)\right],
\end{aligned}
$$

and then, with this at hand, we obtain the first-class Hamiltonian with respect to the constraint $G_{2} \approx 0$

$$
\begin{aligned}
& H_{G U}=H_{G U}^{1}-C_{2}\left[G_{2}, H_{G U}^{1}\right] \\
& +\frac{1}{2} C_{2} C_{2}\left[G_{2}\left[G_{2}, H_{G U}^{1}\right]\right]-\cdots \\
& =H_{G U}^{1}-\int d^{2} x\left[\left(\partial_{i} A^{i}+B_{0}\right) \partial^{j}\left(\pi_{j}+\frac{1}{2 b} \varepsilon_{0 j k} B^{k}\right)\right] .
\end{aligned}
$$

The Hamiltonian gauge algebra relations are given by

$\left[G_{1}, H_{G U}\right]=\left[G_{2}, H_{G U}\right]=0$.

The equations of motion are

$$
\begin{aligned}
\dot{A}_{0}= & -\frac{1}{m} \partial_{i}\left[m A^{i}+\frac{1}{m} \partial^{i}\left(p_{0}+\frac{1}{2 b} \varepsilon_{0 j k} \partial^{j} B^{k}\right)\right], \\
\dot{A}_{i}= & B_{i}-\frac{1}{m^{2}} \partial_{i} \Lambda^{1} \\
\dot{p}^{0}= & -\frac{a}{2} \partial_{i}\left(B^{i}-\partial^{i} A^{0}\right)+\frac{3}{4 b} \varepsilon^{0 i j} \partial_{k} \partial^{k} \partial_{i} A_{j} \\
& +\frac{1}{2} \partial_{i} p^{i}-m^{2} A^{0}+\Lambda^{1}
\end{aligned}
$$




$$
\begin{aligned}
\dot{p}^{i}= & a \partial_{j} \partial^{[j} A^{i]}-\frac{1}{2 b} \varepsilon^{0 i j} \partial_{k} \partial^{k} B_{j}+\frac{1}{b} \varepsilon^{0 i j} \partial_{k} \partial^{k} \partial_{j} A_{0} \\
& -m\left[m A^{i}+\frac{1}{m} \partial^{i}\left(p_{0}+\frac{1}{2 b} \varepsilon_{0 j k} \partial^{j} B^{k}\right)\right] \\
& -\frac{1}{2 b} \varepsilon^{0 i j} \partial_{j} \Lambda^{2}, \\
\dot{B}_{0}= & -\Lambda^{2}+\frac{1}{m^{2}} \partial_{k} \partial^{k} \Lambda^{1}, \\
\dot{B}_{i}= & -a b \varepsilon_{0 i j}\left(B^{j}-\partial^{j} A^{0}\right)-\frac{1}{2} \partial_{k} \partial^{k} A_{i} \\
& -\frac{1}{2} \partial_{i} B_{0}-b \varepsilon_{0 i j} p^{j} \\
& -\partial_{i} \partial^{j}\left[m A_{j}+\frac{1}{m} \partial_{j}\left(p_{0}+\frac{1}{2 b} \varepsilon_{0 k l} \partial^{k} B^{l}\right)\right], \\
\dot{\pi}_{0}= & \frac{1}{2 b} \varepsilon_{0 i j} \partial^{i} B^{j}
\end{aligned}
$$

where $\Lambda^{1}$ and $\Lambda^{2}$ are some arbitrary functions. Under the gauge-fixing conditions

$B_{0}+\partial_{i} A^{i} \approx 0$,

$p_{0}+\frac{1}{2 b} \varepsilon_{0 i j} \partial^{i} B^{j} \approx 0$

$\left(\Lambda^{1}=0\right.$ and $\left.\Lambda^{2}=\partial_{i} B^{i}\right)$, Eqs. (103)-(109) return to the equations of motion for the MECS-Proca model.

The number of physical degrees of freedom of the dynamical system with the phase space locally parameterized by $\left\{A_{\mu}, B_{\mu}, p^{\mu}, \pi^{\mu}\right\}$, subject to the second-class constraints (80) and first-class constraints (95) and (97) is equal to

$$
\begin{aligned}
\overline{\mathscr{N}}_{G U} & =(12 \text { canonical variables }-2 \mathrm{scc}-2 \times 2 \mathrm{fcc}) / 2 \\
& =3=\overline{\mathscr{N}}_{O} .
\end{aligned}
$$

\subsection{Stückelberg coupling}

Based on the equivalence between the first-class system and the original second-class theory, we replace the Hamiltonian path integral of the MECS-Proca model with that of the firstclass system. The Hamiltonian path integral of the first-class system constructed in the above reads

$$
\begin{aligned}
Z= & \int \mathscr{D}\left(A_{\mu}, B_{\mu}, p^{\mu}, \pi^{\mu}, \lambda^{(1)}, \lambda^{(2)}\right) \mu\left(\left[A_{\mu}\right],\left[B_{\mu}\right]\right) \\
& \times \delta\left[\pi_{i}+\frac{1}{2 b} \varepsilon_{0 i j}\left(B^{j}-\partial^{j} A^{0}\right)\right] \\
& \times \operatorname{det}^{1 / 2}\left(\frac{1}{b} \varepsilon_{0 i j} \delta(x-y)\right) \\
& \times \exp \left\{i \int d ^ { 3 } x \left[\left(\partial_{0} A_{\mu}\right) p^{\mu}+\left(\partial_{0} B_{\mu}\right) \pi^{\mu}-\mathscr{H}_{G U}\right.\right. \\
& -\frac{1}{m^{2}} \lambda^{(1)}\left(\partial_{i} p^{i}-m^{2} A_{0}+\partial_{k} \partial^{k} \pi_{0}\right) \\
& \left.\left.-\lambda^{(2)}\left(-\pi_{0}+\frac{1}{2 b} \varepsilon_{0 i j} \partial^{i} A^{j}\right)\right]\right\},
\end{aligned}
$$

where the integration measure ' $\mu\left(\left[A_{\mu}\right],\left[B_{\mu}\right]\right)$ ' includes some suitable canonical gauge conditions. Performing partial integration over the momenta $\pi_{i}$ in the path integral, we arrive at the argument of the exponential in the form

$$
\begin{aligned}
S_{G U}= & \int d^{3} x\left\{\left(\partial_{0} A_{\mu}\right) p^{\mu}+\left(\partial_{0} B_{0}\right) \pi^{0}\right. \\
& \left.-\frac{1}{2 b}\left(\partial_{0} B_{i}\right) \varepsilon^{0 i j}\left(B_{j}-\partial_{j} A_{0}\right)-\frac{a}{4} \partial_{[i} A_{j]}\right]^{[i} A^{j]} \\
& -\frac{a}{2}\left(B_{i}-\partial_{i} A_{0}\right)\left(B^{i}-\partial^{i} A^{0}\right) \\
& +\frac{1}{2 b} \varepsilon_{0 i j}\left(\partial_{k} \partial^{k} A^{0}\right) \partial^{i} A^{j} \\
& +\frac{1}{2 b} \varepsilon_{i 0 j}\left(\partial_{k} \partial^{k} A^{i}\right)\left(B^{j}-\partial^{j} A^{0}\right) \\
& -\frac{1}{2}\left[m A_{i}+\frac{1}{m} \partial_{i}\left(p_{0}+\frac{1}{2 b} \varepsilon_{0 j k} \partial^{j} B^{k}\right)\right] \\
& \times\left[m A^{i}+\frac{1}{m} \partial^{i}\left(p_{0}+\frac{1}{2 b} \varepsilon_{0 l n} \partial^{l} B^{n}\right)\right] \\
& -p^{i} B_{i}-\frac{m^{2}}{2} A_{0} A^{0}+\frac{1}{2 b} B^{0} \varepsilon_{0 j k} \partial^{j} B^{k} \\
& -\frac{1}{m^{2}} \lambda^{(1)}\left(\partial_{i} p^{i}-m^{2} A_{0}+\partial_{k} \partial^{k} \pi_{0}\right) \\
& \left.-\lambda^{(2)}\left(-\pi_{0}+\frac{1}{2 b} \varepsilon_{0 i j} \partial^{i} A^{j}\right)\right\} .
\end{aligned}
$$

Integration over $p^{i}$ leads to a $\delta$ function of the form

$\delta\left(\partial_{0} A_{i}-B_{i}+\frac{1}{m^{2}} \partial_{i} \lambda^{(1)}\right)$,

which permits calculation of the integral over $B_{i}$. Performing a partial integration over the Lagrange multiplier $\lambda^{(2)}$ and $\pi_{0}$, the argument of the exponential becomes

$$
\begin{aligned}
S_{G U}= & \int d^{3} x\left\{\left(\partial_{0} A_{0}\right)\left(p^{0}+\frac{1}{2 b} \varepsilon^{0 i j} \partial_{i} \partial_{0} A_{j}\right)\right. \\
& -\frac{a}{4} \partial_{[i} A_{j]} \partial^{[i} A^{j]}-\frac{a}{2}\left[\partial_{0} A_{i}-\partial_{i}\left(A_{0}-\frac{1}{m^{2}} \lambda^{(1)}\right)\right] \\
& \times\left[\partial^{0} A^{i}-\partial^{i}\left(A^{0}-\frac{1}{m^{2}} \lambda^{(1)}\right)\right] \\
& +\frac{1}{2 b} \varepsilon_{0 i j} \partial_{\lambda} \partial^{\lambda}\left(A^{0}-\frac{1}{m^{2}} \lambda^{(1)}\right) \partial^{i} A^{j} \\
& +\frac{1}{2 b} \varepsilon_{i 0 j}\left(\partial_{\lambda} \partial^{\lambda} A^{i}\right) \partial^{0} A^{j} \\
& +\frac{1}{2 b} \varepsilon_{i j 0}\left(\partial_{\lambda} \partial^{\lambda} A^{i}\right) \partial^{j}\left(A^{0}-\frac{1}{m^{2}} \lambda^{(1)}\right) \\
& -\frac{1}{2}\left[m A_{i}+\frac{1}{m} \partial_{i}\left(p_{0}+\frac{1}{2 b} \varepsilon_{0 j k} \partial^{j} \partial^{0} A^{k}\right)\right] \\
& \times\left[m A^{i}+\frac{1}{m} \partial^{i}\left(p_{0}+\frac{1}{2 b} \varepsilon_{0 l n} \partial^{l} \partial^{0} A^{n}\right)\right] \\
& \left.-\frac{m^{2}}{2} A_{0} A^{0}+\lambda^{(1)} A_{0}\right\} .
\end{aligned}
$$


Using the notation

$\varphi=-\frac{1}{m}\left(p^{0}+\frac{1}{2 b} \varepsilon^{0 i j} \partial_{i} \partial_{0} A_{j}\right), \quad \bar{A}_{0}=A_{0}-\frac{1}{m^{2}} \lambda^{(1)}$,

and integrating over the Lagrange multiplier $\lambda^{(1)}$, the argument of the exponential from the Hamiltonian path integral takes a manifestly Lorentz-covariant form,

$$
\begin{aligned}
S_{G U}= & \int d^{3} x\left[-\frac{a}{4} \partial_{[\mu} \bar{A}_{\nu]} \partial^{[\mu} \bar{A}^{\nu]}\right. \\
& +\frac{1}{2 b} \varepsilon_{\mu \nu \rho}\left(\partial_{\lambda} \partial^{\lambda} \bar{A}^{\mu}\right) \partial^{\nu} \bar{A}^{\rho} \\
& \left.-\frac{1}{2}\left(\partial_{\mu} \varphi-m \bar{A}_{\mu}\right)\left(\partial^{\mu} \varphi-m \bar{A}^{\mu}\right)\right],
\end{aligned}
$$

where $\bar{A}_{\mu}=\left\{\bar{A}_{0}, A_{i}\right\}$, and describes a Stückelberg coupling between the scalar field $\varphi$ and the 1 -form $\bar{A}_{\mu}$. It is obvious that (118) is a higher derivative extension of the result obtained in the previous section (a higher derivative extension involving the CS term). Similar to MCS-Proca model, we find that to the Stückelberg scalar corresponds a combination of the original fields $A_{i}$ and momentum $p^{0}$.

The canonical analysis of the model described by the Lagrangian action (118) displays the constraints (the phase space is locally parameterized by $\left.\left\{A_{\mu}, p^{\mu}, B_{\mu}, \pi^{\mu}, \varphi, p\right\}\right)$

$$
\begin{aligned}
\chi_{i} & \equiv \pi_{i}+\frac{1}{2 b} \varepsilon_{0 i j}\left(B^{j}-\partial^{j} A^{0}\right) \approx 0 \\
G_{1} & \equiv \pi_{0}-\frac{1}{2 b} \varepsilon_{0 i j} \partial^{i} A^{j} \approx 0, \\
G_{2} & \equiv-p_{0}+\partial_{i} \pi^{i} \approx 0, \\
G_{3} & \equiv-\partial_{i} p^{i}+m p-\frac{1}{2 b} \varepsilon_{0 i j} \partial_{k} \partial^{k} \partial^{i} A^{j} \approx 0,
\end{aligned}
$$

and the Hamiltonian

$$
\begin{aligned}
H= & \int d^{2} x\left[\frac{a}{4} \partial_{[i} A_{j]} \partial^{[i} A^{j]}+\frac{a}{2}\left(B_{i}-\partial_{i} A_{0}\right)\left(B^{i}-\partial^{i} A^{0}\right)\right. \\
& -\frac{1}{2 b} \varepsilon_{0 i j}\left(\partial_{k} \partial^{k} A^{0}\right) \partial^{i} A^{j}-\frac{1}{2 b} \varepsilon_{i 0 j}\left(\partial_{k} \partial^{k} A^{i}\right) B^{j} \\
& -\frac{1}{2 b} \varepsilon_{i j 0}\left(\partial_{k} \partial^{k} A^{i}\right) \partial^{j} A^{0}-p^{\mu} B_{\mu}-\frac{1}{2} p^{2} \\
& \left.+m A^{0} p+\frac{1}{2}\left(\partial_{i} \varphi-m A_{i}\right)\left(\partial^{i} \varphi-m A^{i}\right)\right] .
\end{aligned}
$$

The constraints (119) are second-class and the other three constraints are first-class. In order to recover the MECSProca model we chose the gauge conditions

$$
C^{1} \equiv \varphi \approx 0, \quad C^{2} \equiv A_{0} \approx 0, \quad C^{3} \equiv B_{0} \approx 0
$$

such that $\left\{G_{\Delta}, C^{\Delta^{\prime}}\right\}_{\Delta, \Delta^{\prime}=\overline{1,3}}$ form a second-class constraints set and the Hamiltonian path integral is convergent.
The Hamiltonian path integral of the gauge system (118) is given by

$$
\begin{aligned}
Z= & \int \mathscr{D}\left(A_{\mu}, p^{\mu}, B_{\mu}, \pi^{\mu}, \varphi, p\right) \delta\left(\chi_{i}\right) \delta\left(G_{\Delta}\right) \delta\left(C^{\Delta^{\prime}}\right) \\
& \times \exp \left\{i \int d ^ { 3 } x \left[\left(\partial_{0} A_{\mu}\right) p^{\mu}+\left(\partial_{0} B_{\mu}\right) \pi^{\mu}\right.\right. \\
& \left.\left.+\left(\partial_{0} \varphi\right) p-\mathscr{H}\right]\right\} .
\end{aligned}
$$

We integrate over the momenta $\left\{\pi_{i}, \pi_{0}, p_{0}\right\}$ and fields $\left\{\varphi, A_{0}\right\}$ and represent $\delta\left(-\partial_{i} p^{i}+m p-\frac{1}{2 b} \varepsilon_{0 i j} \partial_{k} \partial^{k} \partial^{i} A^{j}\right)$ in the form of an integral functional,

$$
\begin{aligned}
& \int \mathscr{D} \lambda \exp \left\{-i \int d^{3} x \lambda\left(-\partial_{i} p^{i}+m p\right.\right. \\
& \left.\left.-\frac{1}{2 b} \varepsilon_{0 i j} \partial_{k} \partial^{k} \partial^{i} A^{j}\right)\right\} .
\end{aligned}
$$

The path integral takes the form

$$
\begin{aligned}
Z= & \int \mathscr{D}\left(A_{i}, p^{i}, B_{\mu}, p, \lambda\right) \delta\left(C^{3}\right) \exp \left\{i \int d^{3} x\right. \\
& \times\left[\left(\partial_{0} A_{i}\right) p^{i}-\frac{1}{2 b} \varepsilon^{0 i j}\left(\partial_{0} B_{i}\right) B_{j}+\frac{1}{2 b} \varepsilon^{0 i j}\left(\partial^{0} B_{0}\right) \partial_{i} A_{j}\right. \\
& -\frac{a}{4} \partial_{[i} A_{j]} \partial^{[i} A^{j]}-\frac{a}{2} B_{i} B^{i}+\frac{1}{2 b} \varepsilon_{i 0 j}\left(\partial_{k} \partial^{k} A^{i}\right) B^{j} \\
& +\frac{1}{2 b} \varepsilon_{0 i j} B^{0} \partial^{i} B^{j}-p_{i} B^{i}+\frac{1}{2} p^{2}-\frac{m^{2}}{2} A_{i} A^{i} \\
& \left.\left.-\lambda\left(-\partial_{i} p^{i}+m p-\frac{1}{2 b} \varepsilon_{0 i j} \partial_{k} \partial^{k} \partial^{i} A^{j}\right)\right]\right\}
\end{aligned}
$$

Integration over $p^{i}$ leads to a $\delta$ function of the form

$\delta\left(\partial_{0} A_{i}-B_{i}-\partial_{i} \lambda\right)$,

which permits calculation of the integral over $B_{i}$. After integration over the momentum $p$ and field $B_{0}$, the path integral reads

$$
\begin{aligned}
Z= & \int \mathscr{D}\left(A_{i}, \lambda\right) \exp \left\{i \int d ^ { 3 } x \left(-\frac{a}{4} \partial_{[i} A_{j]} \partial^{[i} A^{j]}\right.\right. \\
& -\frac{a}{2}\left(\partial_{0} A_{i}-\partial_{i} \lambda\right)\left(\partial^{0} A^{i}-\partial^{i} \lambda\right) \\
& +\frac{1}{2 b} \varepsilon^{i 0 j}\left(\partial_{\mu} \partial^{\mu} A_{i}\right) \partial_{0} A_{j}+\frac{1}{2 b} \varepsilon^{0 i j}\left(\partial_{\mu} \partial^{\mu} \lambda\right) \partial_{i} A_{j} \\
& \left.\left.+\frac{1}{2 b} \varepsilon^{i j 0}\left(\partial_{\mu} \partial^{\mu} A_{i}\right) \partial_{j} \lambda-\frac{m^{2}}{2} A_{\mu} A^{\mu}\right)\right\} .
\end{aligned}
$$

Using the notation $A_{0}=\lambda$ the argument of the exponential from the Hamiltonian path integral is exactly the MECSProca Lagrangian,

$$
\begin{aligned}
Z= & \int \mathscr{D} A_{\mu} \exp \left\{i \int d ^ { 3 } x \left(-\frac{a}{4} \partial_{[\mu} A_{\nu]} \partial^{[\mu} A^{\nu]}\right.\right. \\
& \left.\left.+\frac{1}{2 b} \varepsilon^{\mu \nu \rho}\left(\partial_{\lambda} \partial^{\lambda} A_{\mu}\right) \partial_{\nu} A_{\rho}-\frac{m^{2}}{2} A_{\mu} A^{\mu}\right)\right\} .
\end{aligned}
$$




\subsection{Chern-Simons coupling}

In the sequel we show that the MECS-Proca model may be related to another first-class theory. Starting from the GU system constructed in the above, subject to the second-class constraints (80), the first-class constraints (95) and (97) and whose evolution is governed by the first-class Hamiltonian (101), we consider the following fields/momenta combinations:

$$
\begin{aligned}
\mathscr{F}_{0} & \equiv A_{0}, \quad \mathscr{F}_{i} \equiv A_{i}+\frac{1}{m^{2}} \partial_{i}\left(p_{0}-\partial_{j} \pi^{j}\right), \\
\mathscr{P}_{i} & \equiv p_{i}-\frac{1}{2 b} \varepsilon_{0 i j} \partial_{k} \partial^{k} A^{j}-\frac{1}{2 b} \varepsilon_{0 i j} \partial^{j} B^{0}, \quad \mathscr{B}_{i} \equiv B_{i},
\end{aligned}
$$

which are in (strong) involution with first-class constraints $G_{a} \approx 0$

$\left[\mathscr{F}_{0}, G_{a}\right]=\left[\mathscr{F}_{i}, G_{a}\right]=\left[\mathscr{P}_{i}, G_{a}\right]=\left[\mathscr{B}_{i}, G_{a}\right]=0$,

and, moreover, $\mathscr{F}_{\mu} \equiv\left\{\mathscr{F}_{0}, \mathscr{F}_{i}\right\}$ is divergenceless on the surface $\chi_{i}^{(1)} \approx 0$

$\partial^{\mu} \mathscr{F}_{\mu}=\mathscr{O}\left(\chi_{i}^{(1)}\right)$.

Similarly to the case of the MCS-Proca model, the first-class Hamiltonian (101) can be written in terms of these quantities

$$
\begin{aligned}
H_{G U}= & \int d^{2} x\left[\frac{a}{4} \partial_{[i} \mathscr{F}_{j]} \partial^{[i} \mathscr{F}^{j]}+\frac{a}{2}\left(\mathscr{B}_{i}-\partial_{i} \mathscr{F}_{0}\right)\right. \\
& \times\left(\mathscr{B}^{i}-\partial^{i} \mathscr{F}^{0}\right)-\frac{1}{2 b} \varepsilon_{0 i j}\left(\partial_{k} \partial^{k} \mathscr{F}^{0}\right) \partial^{i} \mathscr{F}^{j} \\
& -\frac{1}{2 b} \varepsilon_{i j 0}\left(\partial_{k} \partial^{k} \mathscr{F}^{i}\right) \partial^{j} \mathscr{F}^{0}+\frac{m^{2}}{2} \mathscr{F}_{i} \mathscr{F}^{i} \\
& \left.+\frac{m^{2}}{2} \mathscr{F}_{0} \mathscr{F}^{0}+\mathscr{B}^{i} \mathscr{P}_{i}-\left(\partial^{i} \mathscr{F}_{i}\right) \partial^{j} \chi_{j}^{(1)}\right] .
\end{aligned}
$$

Enlarging the phase space by adding the bosonic pairs $\left\{V^{\mu}, P_{\mu}\right\}$, the solution to Eq. (134) takes the form

$\mathscr{F}_{\mu}=-\frac{1}{m} \varepsilon_{\mu \nu \rho} \partial^{v} V^{\rho}$.

When we replace the solution (136) in (95), the constraint takes the form

$\frac{1}{m^{2}}\left(\partial_{i} p^{i}+m \varepsilon_{0 i j} \partial^{i} V^{j}+\partial_{k} \partial^{k} \pi_{0}\right) \approx 0$,

and it remains first-class. Computing the Poisson bracket among the quantity $\partial_{i} p_{0}$ and the first-class constraint (95) and the Poisson bracket between $P_{i}$ and (137), we see that these two quantities are correlated through the relation

$\partial_{i} p_{0}=m \varepsilon_{0 i j} P^{j}$.
Using Eqs. (136) and (138), we write the first-class Hamiltonian as

$$
\begin{aligned}
H_{G U}^{\prime}= & \int d^{2} x\left\{\frac{a}{4} \partial_{[i} A_{j]} \partial^{[i} A^{j]}\right. \\
& +\frac{a}{2}\left[B_{i}+\frac{1}{m} \partial_{i}\left(\varepsilon_{0 j k} \partial^{j} V^{k}\right)\right] \\
& \times\left[B^{i}+\frac{1}{m} \partial^{i}\left(\varepsilon^{0 l n} \partial_{l} V_{n}\right)\right] \\
& +\frac{1}{2 b} \varepsilon_{0 i j} \partial_{k} \partial^{k}\left(\frac{1}{m} \varepsilon^{0 l n} \partial_{l} V_{n}\right) \partial^{i} A^{j} \\
& -\frac{1}{2 b} \varepsilon_{i 0 j}\left(\partial_{k} \partial^{k} A^{i}\right) B^{j}+\frac{1}{2 b} \varepsilon_{i j 0}\left(\partial_{k} \partial^{k} A^{i}\right) \\
& \times \partial^{j}\left(\frac{1}{m} \varepsilon^{0 l n} \partial_{l} V_{n}\right)+\frac{1}{4} \partial^{[i} V^{j]} \partial_{[i} V_{j]} \\
& +\frac{m^{2}}{2}\left(A_{i}+\frac{1}{m} \varepsilon_{0 i j} P^{j}-\frac{1}{m^{2}} \partial_{i} \partial_{j} \pi^{j}\right) \\
& \times\left(A^{i}+\frac{1}{m} \varepsilon^{0 i l} P_{l}-\frac{1}{m^{2}} \partial^{i} \partial^{l} \pi_{l}\right) \\
& -\partial^{i}\left(A_{i}+\frac{1}{m} \varepsilon_{0 i k} P^{k}-\frac{1}{m^{2}} \partial_{i} \partial_{k} \pi^{k}\right) \\
& \times \partial^{j}\left(\pi \pi_{j}+\frac{1}{2 b} \varepsilon_{0 j k} B^{k}\right) \\
& \left.-\frac{1}{2 b} \varepsilon_{0 j k} B^{0} \partial^{j} B^{k}+p^{i} B_{i}\right\} .
\end{aligned}
$$

If we count the number of physical degrees of freedom of the system with the phase space locally parameterized by $\left\{A_{i}, B_{\mu}, V^{\mu}, p^{i}, \pi^{\mu}, P_{\mu}\right\}$ subject to the second-class constraints (80), first-class constraints (97) and (137) and whose evolution is governed by the first-class Hamiltonian (139), we obtain

$$
\begin{aligned}
\overline{\mathscr{N}}_{G U}^{\prime} & =(16 \text { canonical variables }-2 \mathrm{scc}-2 \times 2 \mathrm{fcc}) / 2 \\
& =5 \neq \overline{\mathscr{N}}_{G U} .
\end{aligned}
$$

Imposing the first-class constraints

$-\partial^{i} P_{i} \approx 0, \quad P_{0} \approx 0$,

the number of physical degrees of freedom is conserved

$$
\begin{aligned}
\overline{\mathscr{N}}_{G U}^{\prime} & =(16 \text { canonical variables }-2 \mathrm{scc}-2 \times 4 \mathrm{fcc}) / 2 \\
& =3=\overline{\mathscr{N}}_{G U} .
\end{aligned}
$$

For each first-class theory, derived in the above, we are able to identify a set of fundamental classical observables such that they are in one-to-one correspondence and they possess the same Poisson brackets. Since the number of physical degrees of freedom is the same for both theories and the corresponding algebras of classical observables are isomorphic, the previously exposed procedure preserves the equivalence between the two first-class theories. As a result, the GU and 
the first-class system remain equivalent also at the level of the Hamiltonian path integral quantization. This further implies that the first-class system is completely equivalent with the MECS-Proca model. Due to this equivalence we can replace the Hamiltonian path integral of the MECS-Proca model with the one associated with the first-class system,

$$
\begin{aligned}
Z^{\prime}= & \int \mathscr{D}\left(A_{i}, B_{\mu}, V^{\mu}, p^{i}, \pi^{\mu}, P_{\mu}, \lambda^{\prime} s\right) \\
& \times \mu\left(\left[A_{i}\right],\left[B_{\mu}\right],\left[V^{\mu}\right]\right) \\
& \times \delta\left[\pi_{i}+\frac{1}{2 b} \varepsilon_{0 i j}\left(B^{j}+\frac{1}{m} \varepsilon^{0 k l} \partial^{j} \partial_{k} V_{l}\right)\right] \\
& \times \operatorname{det}^{1 / 2}\left(\frac{1}{b} \varepsilon_{0 i j} \delta(x-y)\right) \exp \left\{i \int d ^ { 3 } x \left[\left(\partial_{0} A_{i}\right) p^{i}\right.\right. \\
& +\left(\partial_{0} B_{\mu}\right) \pi^{\mu}+\left(\partial_{0} V^{\mu}\right) P_{\mu}-\mathscr{H}_{G U}^{\prime} \\
& -\frac{1}{m^{2}} \lambda^{(1)}\left(\partial_{i} p^{i}+m \varepsilon_{0 i j} \partial^{i} V^{j}+\partial_{k} \partial^{k} \pi_{0}\right) \\
& -\lambda^{(2)}\left(-\pi_{0}+\frac{1}{2 b} \varepsilon_{0 i j} \partial^{i} A^{j}\right) \\
& \left.\left.+\lambda^{(3)} \partial^{i} P_{i}-\lambda^{(4)} P_{0}\right]\right\} .
\end{aligned}
$$

After a partial integration over the momenta $\pi_{i}$ in the path integral, the argument of the exponential reads

$$
\begin{aligned}
& S_{G U}^{\prime}=\int d^{3} x\left\{\left(\partial_{0} A_{i}\right) p^{i}+\left(\partial_{0} B_{0}\right) \pi^{0}+\left(\partial_{0} V^{\mu}\right) P_{\mu}\right. \\
& +\frac{1}{2 b}\left(\partial_{0} B_{i}\right) \varepsilon^{0 i j}\left[-B_{j}-\partial_{j}\left(\frac{1}{m} \varepsilon^{0 k l} \partial_{k} V_{l}\right)\right] \\
& -\frac{a}{4} \partial_{[i} A_{j]} \partial^{[i} A^{j]}-\frac{a}{2}\left[B_{i}+\partial_{i}\left(\frac{1}{m} \varepsilon_{0 j k} \partial^{j} V^{k}\right)\right] \\
& \times\left[B^{i}+\partial^{i}\left(\frac{1}{m} \varepsilon^{0 \ln } \partial_{l} V_{n}\right)\right] \\
& -\frac{1}{2 b} \varepsilon_{0 i j} \partial_{k} \partial^{k}\left(\frac{1}{m} \varepsilon^{0 l n} \partial_{l} V_{n}\right) \partial^{i} A^{j} \\
& +\frac{1}{2 b} \varepsilon_{i 0 j}\left(\partial_{k} \partial^{k} A^{i}\right) B^{j}-\frac{1}{2 b} \varepsilon_{i j 0}\left(\partial_{k} \partial^{k} A^{i}\right) \\
& \times \partial^{j}\left(\frac{1}{m} \varepsilon^{0 \ln } \partial_{l} V_{n}\right)-\frac{1}{4} \partial^{[i} V^{j]} \partial_{[i} V_{j]} \\
& -\frac{m^{2}}{2}\left[A_{i}+\frac{1}{m} \varepsilon_{0 i j} P^{j}+\frac{1}{m^{2}} \partial_{i}\left(\frac{1}{2 b} \varepsilon_{0 j k} \partial^{j} B^{k}\right)\right] \\
& \times\left[A^{i}+\frac{1}{m} \varepsilon^{0 i l} P_{l}+\frac{1}{m^{2}} \partial_{i}\left(\frac{1}{2 b} \varepsilon_{0 l n} \partial^{l} B^{n}\right)\right] \\
& +\frac{1}{2 b} \varepsilon_{0 j k} B^{0} \partial^{j} B^{k}-p^{i} B_{i} \\
& -\frac{1}{m^{2}} \lambda^{(1)}\left(\partial_{i} p^{i}+m \varepsilon_{0 i j} \partial^{i} V^{j}+\partial_{k} \partial^{k} \pi_{0}\right) \\
& -\lambda^{(2)}\left(-\pi_{0}+\frac{1}{2 b} \varepsilon_{0 i j} \partial^{i} A^{j}\right) \\
& \left.+\lambda^{(3)} \partial^{i} P_{i}-\lambda^{(4)} P_{0}\right\} \text {. }
\end{aligned}
$$

Integration over $p^{i}$ leads to a $\delta$ function of the form

$\delta\left(\partial_{0} A_{i}-B_{i}+\frac{1}{m^{2}} \partial_{i} \lambda^{(1)}\right)$,

which permits calculation of the integral over $B_{i}$. Performing a partial integration over the field $V_{0}$, momenta $\left\{\pi_{0}, P_{0}, P_{i}\right\}$, and Lagrange multipliers $\left\{\lambda^{(2)}, \lambda^{(4)}\right\}$, the argument of the exponential from the Hamiltonian path integral reads

$$
\begin{aligned}
S_{G U}^{\prime}= & \int d^{3} x\left\{-\frac{a}{4} \partial_{[i} A_{j]} \partial^{[i} A^{j]}\right. \\
& -\frac{a}{2}\left[\partial_{0} A_{i}+\partial_{i}\left(\frac{1}{m^{2}} \lambda^{(1)}+\frac{1}{m} \varepsilon_{0 j k} \partial^{j} V^{k}\right)\right] \\
& \times\left[\partial^{0} A^{i}+\partial^{i}\left(\frac{1}{m^{2}} \lambda^{(1)}+\frac{1}{m} \varepsilon^{0 l n} \partial_{l} V_{n}\right)\right] \\
& -\frac{1}{2 b} \varepsilon_{0 i j} \partial_{\lambda} \partial^{\lambda}\left(\frac{1}{m^{2}} \lambda^{(1)}+\frac{1}{m} \varepsilon^{0 k l} \partial_{k} V_{l}\right) \partial^{i} A^{j} \\
& +\frac{1}{2 b} \varepsilon_{i 0 j}\left(\partial_{\lambda} \partial^{\lambda} A^{i}\right) \partial^{0} A^{j}-\frac{1}{2 b} \varepsilon_{i j 0}\left(\partial_{\lambda} \partial^{\lambda} A^{i}\right) \\
& \times \partial^{j}\left(\frac{1}{m^{2}} \lambda^{(1)}+\frac{1}{m} \varepsilon^{0 k l} \partial_{k} V_{l}\right)+\frac{1}{4} \partial_{[i} V_{j]} \partial^{[i} V^{j]} \\
& +\frac{1}{2}\left(\partial_{0} V_{i}-\partial_{i} \lambda^{(3)}\right)\left(\partial^{0} V^{i}-\partial^{i} \lambda^{(3)}\right) \\
& -m \varepsilon_{0 i j}\left(\frac{1}{m^{2}} \lambda^{(1)}+\frac{1}{m} \varepsilon^{0 k l} \partial_{k} V_{l}\right)\left(\partial^{i} V^{j}\right) \\
& \left.+m \varepsilon_{i 0 j} A^{i}\left(\partial^{0} V^{j}-\partial^{j} \lambda^{(3)}\right)\right\} .
\end{aligned}
$$

Using the notations

$\bar{A}_{0}=-\left(\frac{1}{m^{2}} \lambda^{(1)}+\frac{1}{m} \varepsilon_{0 j k} \partial^{j} V^{k}\right), \quad \bar{V}_{0}=\lambda^{(3)}$,

the argument of the exponential from the Hamiltonian path integral takes a manifestly Lorentz-covariant form,

$$
\begin{aligned}
S_{G U}^{\prime}= & \int d^{3} x\left[-\frac{a}{4} \partial_{[\mu} \bar{A}_{\nu]} \partial^{[\mu} \bar{A}^{\nu]}\right. \\
& +\frac{1}{2 b} \varepsilon_{\mu \nu \rho}\left(\partial_{\lambda} \partial^{\lambda} \bar{A}^{\mu}\right) \partial^{\nu} \bar{A}^{\rho}+\frac{1}{4} \partial_{[\mu} \bar{V}_{\nu]} \partial^{[\mu} \bar{V}^{\nu]} \\
& \left.+m \varepsilon_{\mu \nu \rho} \bar{A}^{\mu} \partial^{\nu} \bar{V}^{\rho}\right],
\end{aligned}
$$

where $\bar{A}_{\mu}=\left\{\bar{A}_{0}, A_{i}\right\}$ and $\bar{V}_{\mu}=\left\{\bar{V}_{0}, V_{i}\right\}$. The above functional describes a CS coupling between the 1 -form $\bar{A}_{\mu}$ and the 1 -form $\bar{V}_{\mu}$, and it is a higher derivative extension of the functional (45).

\section{Conclusions}

In this paper, the MCS-Proca model has been analyzed from the point of view of the Hamiltonian path integral quantiza- 
tion, in the framework of gauge-unfixing approach. The same quantization procedure was applied to a higher order derivative extension of MCS-Proca model. The first step of this approach is represented by the construction of an equivalent first-class system. In order to construct the equivalent firstclass system with the MECS-Proca model, we performed a partial gauge-unfixing (we maintained the second-class constraints (80)); meanwhile, in the case of the MCS-Proca model, we accomplished a total gauge-unfixing. Both models did not require extensions of the original phase space in order to construct the equivalent first-class systems. The second step involved the construction of the Hamiltonian path integral corresponding to the equivalent first-class system for each model. The Hamiltonian path integral of the first-class systems took a manifestly Lorentz-covariant form, after integrating out the auxiliary fields and performing some field redefinitions. Starting from the Hamiltonian path integral of the equivalent non-higher derivative first-class system, we arrived at the Lagrangian path integral corresponding to Stückelberg coupling between a scalar field and a 1-form or for an appropriate phase space extension we identified the Lagrangian path integral for two kinds of 1 -forms with CS coupling (a non-higher order derivative term). The results obtained in the case of MECS-Proca model are higher derivative extensions (involving the CS term) of the results obtained in the case of MCS-Proca model.

Acknowledgments The author wishes to thank E. M. Cioroianu for useful discussions and comments. I am very grateful to Prof. S. Deser for calling my attention to Ref. [43]. Also, I would like to thank to referees for bringing to my attention Ref. [44].

Open Access This article is distributed under the terms of the Creative Commons Attribution 4.0 International License (http://creativecomm ons.org/licenses/by/4.0/), which permits unrestricted use, distribution, and reproduction in any medium, provided you give appropriate credit to the original author(s) and the source, provide a link to the Creative Commons license, and indicate if changes were made.

Funded by SCOAP ${ }^{3}$.

\section{References}

1. T.J. Allen, M.J. Bowick, A. Lahiri, Topological mass generation in 3+1 dimensions. Mod. Phys. Lett. A 6, 559 (1991)

2. A.S. Vytheeswaran, Gauge unfixing in second class constrained systems. Ann. Phys. 236, 297 (1994)

3. E.B. Park, Y.W. Kim, Y.J. Park, Y. Kim, W.T. Kim, Batalin-Tyutin quantization of the Chern-Simons-Proca theory. Mod. Phys. Lett. A 10, 1119 (1995). arXiv:hep-th/9504151

4. N. Banerjee, R. Banerjee, S. Ghosh, Quantization of second class systems in the Batalin-Tyutin formalism. Ann. Phys. 241, 237 (1995). arXiv:hep-th/9403069

5. H. Sawayanagi, Hamiltonian BRST quantization of an Abelian massive vector field with an antisymmetric tensor field. Mod. Phys. Lett. A 10, 813 (1995)

6. C. Bizdadea, S.O. Saliu, The BRST quantization of massive abelian two-form gauge fields. Phys. Lett. B 368, 202 (1996)
7. C. Bizdadea, Some remarks on the BRST quantization of massive Abelian two-form gauge fields. Phys. Rev. D 53, 7138 (1996)

8. C. Bizdadea, The hamiltonian BRST quantization of massive abelian p-form gauge fields. J. Phys. A: Math. Gen. 29, 3985 (1996)

9. N. Banerjee, R. Banerjee, Generalized Hamiltonian embedding of the Proca model. Mod. Phys. Lett. A 11, 1919 (1996). arXiv:hep-th/9511212

10. Y.W. Kim, M.I. Park, Y.J. Park, S.J. Yoon, BRST quantization of the Proca model based on the BFT and the BFV formalism. Int. J. Mod. Phys. A 12, 4217 (1997). arXiv:hep-th/9702002

11. A.S. Vytheeswaran, Gauge invariances in the Proca model. Int. J. Mod. Phys. A 13, 765 (1998). arXiv:hep-th/9701050

12. S.T. Hong, Y.W. Kim, Y.J. Park, K.D. Rothe, Symplectic embedding and Hamilton-Jacobi analysis of Proca model. Mod. Phys. Lett. A 17, 435 (2002). arXiv:hep-th/0112170

13. H. Ruegg, M. Ruiz-Altaba, The Stueckelberg field. Int. J. Mod. Phys. A 19, 3265 (2004). arXiv:hep-th/0304245

14. E.M. Cioroianu, S.C. Sararu, O. Balus, First-class approaches to massive 2-forms. Int. J. Mod. Phys. A 25, 185 (2010). arXiv: 1001.5146

15. E.M. Cioroianu, Note on the dynamics of a pseudo-classical spinning particle. Mod. Phys. Lett. A 26, 589 (2011)

16. S.C. Sararu, Massive p-forms: first-class approaches. Int. J. Mod. Phys. A 27, 1250119 (2012)

17. S.C. Sararu, On covariant quantization of the massive self-dual 3-forms in 7 dimensions. Int. J. Theor. Phys. 51, 2623 (2012)

18. S.C. Sararu, From massive self-dual p-forms towards gauge pforms. Cent. Eur. J. Phys. 11, 59 (2013)

19. K. Harada, H. Mukaida, Gauge invariance and systems with second class constraints. Z. Phys. C 48, 151 (1990)

20. P. Mitra, R. Rajaraman, New results on systems with second-class constraints. Ann. Phys. 203, 137 (1990)

21. M. Henneaux, C. Teitelboim, Quantization of Gauge Systems (Princeton University Press, Princeton, 1992)

22. R. Anishettyt, A.S. Vytheeswaran, Gauge invariance in secondclass constrained systems. J. Phys. A: Math. Gen. 26, 5613 (1993)

23. C. Bizdadea, S.O. Saliu, The BRST quantization of second-class constrained systems. Nucl. Phys. B 456, 473 (1995)

24. L.D. Faddeev, S.L. Shatashvili, Realization of the Schwinger term in the Gauss law and the possibility of correct quantization of a theory with anomalies. Phys. Lett. B 167, 225 (1986)

25. I.A. Batalin, E.S. Fradkin, Operator quantization of dynamical systems with irreducible first- and second-class constraints. Phys. Lett. B 180, 157 (1986)

26. I.A. Batalin, E.S. Fradkin, Operational quantization of dynamical systems subject to second-class constraints. Nucl. Phys. B 279, 514 (1987)

27. I.A. Batalin, I.V. Tyutin, Existence theorem for the effective gauge algebra in the generalized canonical formalism with Abelian conversion of second-class constraints. Int. J. Mod. Phys. A 6, 3255 (1991)

28. S. Deser, R. Jackiw, "Self-duality" of topologically massive gauge theories. Phys. Lett. B 139, 371 (1984)

29. P.K. Townsend, K. Pilch, P. van Nieuwenhuizen, Self-duality in odd dimensions. Phys. Lett. B 136, 38 (1984)

30. S. Deser, R. Jackiw, P. van Nieuwenhuizen, Three-dimensional massive gauge theories. Phys. Rev. Lett. 48, 975 (1982)

31. S. Deser, R. Jackiw, S. Templeton, Topologically massive gauge theories. Ann. Phys. 140, 372 (1982)

32. R. Banerjee, H.J. Rothe, K.D. Rothe, Equivalence of the MaxwellChern-Simons theory and a self-dual model. Phys. Rev. D 52, 3750 (1995). arXiv:hep-th/9504067

33. R. Banerjee, H.J. Rothe, K.D. Rothe, Hamiltonian embedding of the self-dual model and equivalence with Maxwell-Chern-Simons theory. Phys. Rev. D 55, 6339 (1997). arXiv:hep-th/9611077 
34. R. Banerjee, H.J. Rothe, Batalin-Fradkin-Tyutin embedding of a self-dual model and the Maxwell-Chern-Simons theory. Nucl. Phys. B 447, 183 (1995). arXiv:hep-th/9504066

35. L. Heisenberg, Generalization of the Proca action. JCAP 05, 015 (2014). arXiv: 1402.7026

36. S. Deser, R. Jackiw, Higher derivative Chern-Simons extensions. Phys. Lett. B 451, 73 (1999). arXiv:hep-th/9901125

37. A. de Souza Dutra, C.P. Natividade, Class of self-dual models in three dimensions. Phys. Rev. D 61, 027701 (2000). arXiv:hep-th/0002114

38. D. Bazeia, R. Menezes, J.R. Nascimento, R.F. Ribeiro, C. Wotzasek, Dual equivalence in models with higher-order derivatives. J. Phys. A 36, 9943 (2003). arXiv:hep-th/0210311

39. A. Accioly, M. Diase, Algorithm for probing the unitarity of topologically massive models. Int. J. Theor. Phys. 44, 1123 (2005). arXiv:hep-th/0511242

40. A. Accioly, M. Diase, Is it physically sound to add a topologically massive term to three-dimensional massive electromagnetic or gravitational models? Int. J. Mod. Phys. A 21, 559 (2006). arXiv:hep-th/0507186

41. B. Podolsky, A generalized electrodynamics part I-non-quantum. Phys. Rev. 62, 68 (1942)

42. C. Pinheiro, G.O. Pires, N. Tomimura, Some quantum aspects of three-dimensional Einstein-Chern-Simons-Proca massive gravity. Nuovo Cim. B 111, 1023 (1996). arXiv:gr-qc/9704004

43. S. Deser, B. Tekin, Massive, topologically massive, models. Class. Quant. Grav. 19, L97 (2002). arXiv:hep-th/0203273

44. R. Banerjee, S. Kumar, Self-dual models and mass generation in planar field theory. Phys. Rev. D 63, 125008 (2001). arXiv:hep-th/0007148

45. R. Banerjee, B. Chakraborty, T. Scaria, Polarization vectors, doublet structure and Wigner's little group in planar field theory. Int. J. Mod. Phys. A 16, 3967 (2001). arXiv:hep-th/0011011

46. M.V. Ostrogradsky, Memoires sur les equations differentielles relatives au probleme des isoperimetres. Mem. Ac. St. Petersbourg VI, 385 (1850)

47. D.M. Gitman, S.L. Lyakhovich, I.V. Tyutin, Canonical quantization of the Yang-Mills lagrangian with higher derivatives. Sov. Phys. J. 28, 554 (1985)
48. D.M. Gitman, I.V. Tyutin, Quantization of Fields with Constraints (Springer-Verlag, Berlin, Heidelberg, 1990)

49. V.V. Nesterenko, Singular Lagrangians with higher derivatives. J. Phys. A 22, 1673 (1989)

50. S. Kumar, Lagrangian and Hamiltonian formulations of higher order Chern-Simons theories. Int. J. Mod. Phys. A 18, 1613 (2003). arXiv:hep-th/0112121

51. C.M. Reyes, Testing symmetries in effective models of higher derivative field theories. Phys. Rev. D 80, 105008 (2009). arXiv:0901.1341

52. R. Banerjee, P. Mukherjee, B. Paul, Gauge symmetry and Walgebra in higher derivative systems. J. High Energy Phys. 08, 085 (2011). arXiv:1012.2969

53. M.S. Plyushchay, Massive relativistic point particle with rigidity. Int. J. Mod. Phys. A 4, 3851 (1989)

54. M.S. Plyushchay, The model of the relativistic particle with torsion. Nucl. Phys. B 362, 54 (1991)

55. P. Mukherjee, B. Paul, Gauge invariances of higher derivative Maxwell-Chern-Simons field theory: a new Hamiltonian approach. Phys. Rev. D 85, 045028 (2012). arXiv:1111.0153

56. B. Paul, Gauge symmetry and Virasoro algebra in quantum charged rigid membrane: a first order formalism. Phys. Rev. D 87, 045003 (2013). arXiv:1212.5902

57. R. Banerjee, P. Mukherjee, B. Paul, New Hamiltonian analysis of Regge-Teitelboim minisuperspace cosmology. Phys. Rev. D 89, 043508 (2014). arXiv: 1307.4920

58. P. Dirac, Generalized Hamiltonian dynamics. Can. J. Math. 2, 129 (1950)

59. P. Dirac, Lectures on Quantum Mechanics (Academic Press, New York, 1967)

60. R. Ferraro, M. Henneaux, M. Puchin, On the quantization of reducible gauge systems. J. Math. Phys. 34, 2757 (1993). arXiv:hep-th/9210070

61. E.C.G. Stueckelberg, Interaction energy in electrodynamics and in the field theory of nuclear forces. Helv. Phys. Acta 11, 225 (1938)

62. C. Bizdadea, E.M. Cioroianu, S.O. Saliu, Irreducible Hamiltonian BRST approach to topologically coupled abelian forms. Phys. Scr. 60, 120 (1999). arXiv:hep-th/9912201 